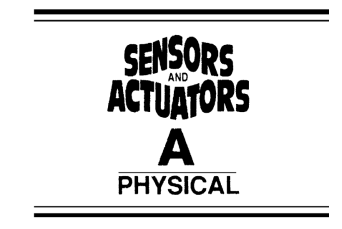

www.elsevier.com/locate/sna

\title{
Enhanced short wavelength response in laser-scanned-photodiode image sensor using an a-SiC:H/a-Si:H tandem structure
}

\author{
A. Fantoni ${ }^{\mathrm{a}, \mathrm{b}, *}$, P. Louro ${ }^{\mathrm{a}}$, M. Fernandes ${ }^{\mathrm{a}}$, M. Vieira ${ }^{\mathrm{a}}$, \\ G. Lavareda ${ }^{b}$, C. Nunes De Carvalho ${ }^{b}$ \\ a ISEL-DEETC, Rua Conselheiro Emidio Navarro, 1949-014 Lisboa, Portugal \\ b FCT-UNL, Quinta da Torre, 2825 Monte de Caparica, Portugal
}

Received 13 September 2004; received in revised form 31 March 2005; accepted 26 April 2005

Available online 11 July 2005

\begin{abstract}
We report in this paper the recent advances we obtained in optimizing a color image sensor based on the laser-scanned-photodiode (LSP) technique. A novel device structure based on a a-SiC:H/a-Si:H pin/pin tandem structure has been tested for a proper color separation process that takes advantage on the different filtering properties due to the different light penetration depth at different wavelengths a-Si:H and a$\mathrm{SiC}: \mathrm{H}$. While the green and the red images give, in comparison with previous tested structures, a weak response, this structure shows a very good recognition of blue color under reverse bias, leaving a good margin for future device optimization in order to achieve a complete and satisfactory RGB image mapping. Experimental results about the spectral collection efficiency are presented and discussed from the point of view of the color sensor applications. The physics behind the device functioning is explained by recurring to a numerical simulation of the internal electrical configuration of the device.
\end{abstract}

(C) 2005 Elsevier B.V. All rights reserved.

Keywords: a-Si:H; Numerical simulation; Heterostructures; Tandem cell

\section{Introduction}

A black and white image sensor based on the measure of the photocurrent perturbation caused by a laser spot over an image projected on the device surface has been recently presented. Called laser scanned photodiode (LSP) this sensor has recently shown its potential capability as monochrome image sensing devices $[1,2]$. The LSP under short circuit is naturally monochromatic.

For simultaneous light intensity and color detection, the wavelength filtering property of a-SiC:H (variation of the absorption coefficient with wavelength) can be combined with the asymmetric behavior of the photocurrent profile we observed in tandem structures. The wavelength of a laser scanner can be tuned to compensate the asymmetry in the

\footnotetext{
* Corresponding author. Tel.: +35121831 7289; fax: +351218317114. E-mail address: afantoni@deetc.isel.ipl.pt (A. Fantoni).
}

photogeneration profile, producing a peak on the resulting photocurrent. Once that a B\&W image has been detected, a green scanner can be used to detect red color while a red scanner detects the green radiation. A similar technique was proposed for an a-Si:H p-i-n structure with carbon in the doped layers, where the photocurrent were measured under forward bias condition [3].

Our simulation results pointed out in our past work that further improvement in the color recognition process can be obtained with a $\mathrm{p}-\mathrm{i}-\mathrm{n}(\mathrm{a}-\mathrm{SiC}: \mathrm{H}) / \mathrm{p}-\mathrm{i}-\mathrm{n}(\mathrm{a}-\mathrm{Si} \mathrm{H})$ structure[4]. By using a-SiC:H in the top cell, the red part of the image spectrum is only absorbed in the bottom cell, while the blue one remain confined within the top cell. The electrical internal configuration assumes different profiles depending on the color of the incident light. The thickness of the two sub-cells must be optimized in order to separate the blue absorption in the top cell and the red in the bottom one, thus avoiding the overlapping of the absorption profiles. 
We present in this work experimental results about the spectral response of the pin $(\mathrm{a}-\mathrm{SiC}: \mathrm{H}) / \mathrm{pin}(\mathrm{a}-\mathrm{Si}: \mathrm{H})$ tandem cell, measured under different external polarization. In order to explain the physics of the device operation, results obtained by a numerical simulation of the internal electric configuration are also presented and compared.

\section{Experimental set-up}

We have characterized and simulated a tandem pinpin device with the following structure deposited with PECVD technique: ITO/p-type a-SiC:H $(20 \mathrm{~nm}) / \mathrm{a}-$ SiC:H $(200 \mathrm{~nm}) /$ n-type a-SiC:H $(20 \mathrm{~nm}) /$ p-type a-SiC:H $(20 \mathrm{~nm})) / \mathrm{a}-\mathrm{Si}: \mathrm{H}(500 \mathrm{~nm}) / \mathrm{n}$-type a-Si:H (50 nm)/ITO. Fig. 1 schematically shows the structure under analysis. Deposition temperature and RF power were $300^{\circ} \mathrm{C}$ and $20 \mathrm{~W}$, respectively.

Carbon concentration (introduced through a flux of $\mathrm{CH}_{4}$ during the deposition) in the top a-SiC:H cell produces an optical gap of about $2.0 \mathrm{eV}$. The purpose of using a wide band gap material, such as a-SiC:H in the front photodiode is the enhancement of the blue sensitivity and of the red transmittance. Doping level in the internal recombination junction is about half the doping level of the external $p$ and n-layers. More technical details about film characteristics and deposition conditions can be found elsewhere [5].

For a better color screening, the image is projected over the top surface, and it is absorbed by the structure through the thin a-SiC:H cell, while the laser scanner is absorbed through the bottom thick a-Si:H cell. Transparent contacts on both the surfaces are required by the LSP photocurrent measurement.

The thick back photodiode controls the conversion efficiency in the red spectral range, while the thin one controls it in the blue range. A modulated light beam shines on the sample through the thick diode and a steady state bias light was applied through the back side. The photocurrent generated by the probe beam was then measured by a lockin amplifier while varying the electrical bias in the range $(-6$ to $+2 \mathrm{~V})$. The light probe with wavelength $\lambda_{\mathrm{s}}=650 \mathrm{~nm}$ and power flux $\Phi_{\mathrm{s}}=5.5 \mu \mathrm{W} \mathrm{cm}{ }^{-2}$ was generated by a laser, while the steady state bias light was obtained by filtering a halogen light source with bandpass filters: $F W H M=0 \mathrm{~nm}$, $1 \lambda_{\mathrm{L}}=450 \mathrm{~nm}$ (blue), $550 \mathrm{~nm}$ (green), $650 \mathrm{~nm}$ (red), incident power $0<\phi_{\mathrm{L}}<200 \mu \mathrm{W} / \mathrm{cm}^{2}$.

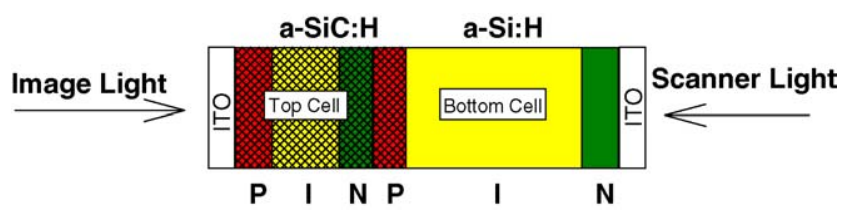

Fig. 1. Schematic representation of the tandem structure under analysis.

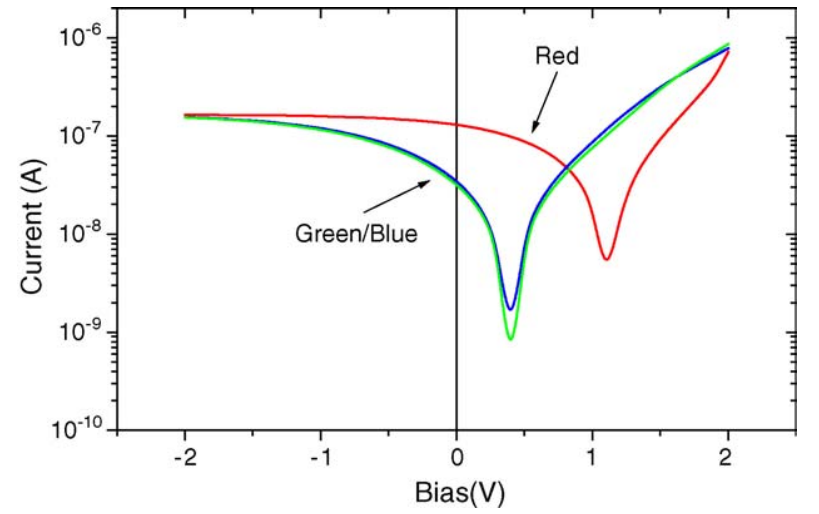

Fig. 2. I-V curve under red $(650 \mathrm{~nm})$, blue $(450 \mathrm{~nm})$ and green $(550 \mathrm{~nm})$ illumination. Light entering through the bottom a-Si:H thick cell. Incident power: $220 \mu \mathrm{W} \mathrm{cm}^{-2}$.

\section{Results and discussion}

Figs. 2 and 3 show the I-V measurements obtained by illuminating the cell, respectively, from the bottom and the top surface. The current values are very small, due to the low conductivity of the a-SiC:H layers and to the unbalanced photo-generation profile between the two sub-cells. When the device is illuminated from the bottom thick cell, the current under blue and green radiations presents a very similar dependence from the applied bias. The open circuit voltage (Voc) is very small, revealing that internal transport of photogenerated carriers is not completely activated. Under red light, the photo-generation is almost uniform (and restricted) within the bottom cell, the current value remains low, but the Voc reaches a stable value of $1.2 \mathrm{~V}$, which falls close to the Voc of $1.5 \mathrm{~V}$ we observed under white light, showing that the internal transport mechanism is activated by the red radiation. When the structure is illuminated through the thin top a-SiC:H cell, the I-V characteristic remains almost unchanged under red light irradiation. As expected, the red light photo-generation within the a-SiC:H cell is almost null, while within the thick a-Si:H the photogeneration profile is almost uniform, independently

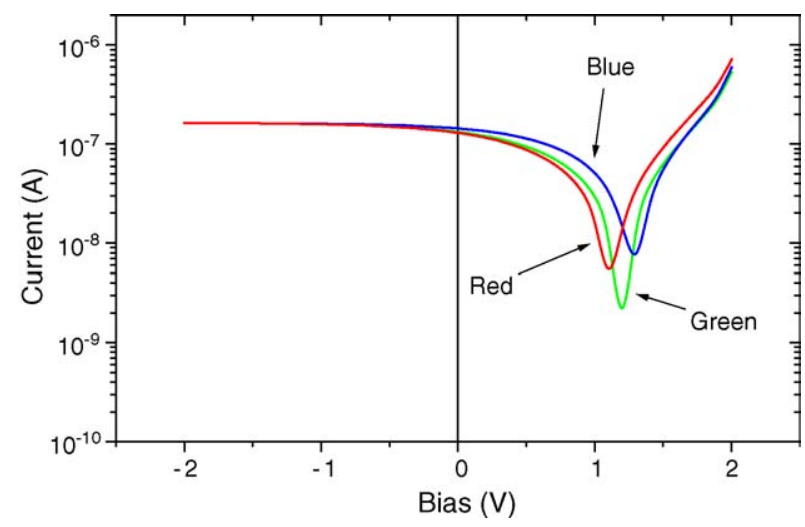

Fig. 3. I-V curve under red $(650 \mathrm{~nm})$, blue $(450 \mathrm{~nm})$ and green $(550 \mathrm{~nm})$ illumination. Light entering through the top a-SiC: $\mathrm{H}$ thin cell. Incident power: $220 \mu \mathrm{W} \mathrm{cm}{ }^{-2}$. 
on the illuminated side. Under blue radiation, the top cell plays an active role but the photogeneration does not reach the bottom cell. An intermediary situation is observed under green illumination, where the top cell is completely depleted, and photogeneration process reaches also the bottom cell. The Voc becomes similar to the one observed under red light. The current value remains low, but it is to be remarked that high values of the current, even if welcome for measuring simplicity, are not required for color sensor applications. We can conclude from the analysis of these two figures that the carrier transport mechanism is activated when the cell is illuminated from the top a-SiC:H cell, while only the red radiation succeeds in activating the cell when light shines on the bottom a-Si:H cell.

Fig. 4 reports the spectral response of the device measured through the bottom a-Si:H thick cell, with and without background bias light entering through the top a-SiC:H thin cell. Independently on the wavelength of the background bias light, the maximum responsivity is obtained at a wavelength of about $650 \mathrm{~nm}$, i.e. in the red part of the spectrum, as could be expected by the previous analysis of the I-V characteristics presented in Fig. 2. It is interesting to note that with background blue bias light through the top a-SiC:H cell the spectral response is enhanced in relation to the corresponding SR without bias light. In opposition, the application of both red and green bias light causes the responsivity of the cell to decrease. This consideration leads us to conclude that, even under short-circuit condition it is possible to separate blue colour from red and green, by a simple analysis of the algebraic sign of the scanner perturbation of the measured photocurrent.

The spectral response (SR) without bias light and under red bias light is independent on the applied external bias, a small dependence has been observed under green bias light conditions. Fig. 5 shows the variation of the spectral response with an external applied bias varying between -2 and $2 \mathrm{~V}$ under blue bias light conditions. The application of a for-

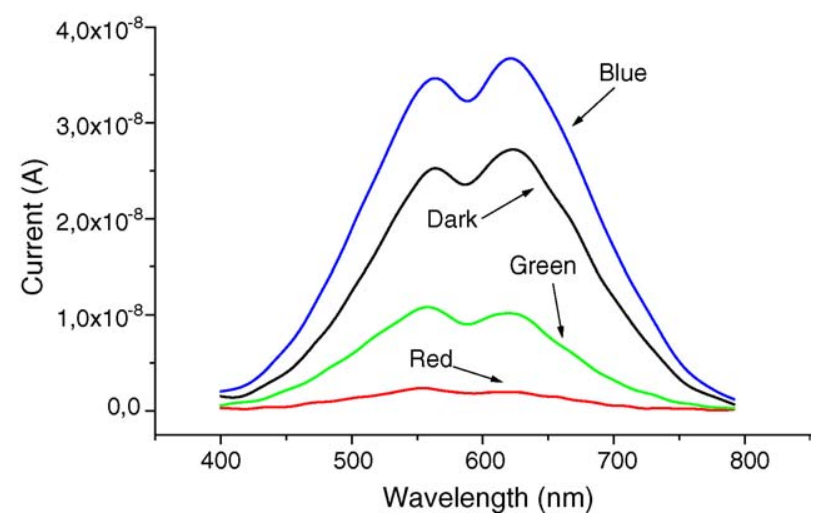

Fig. 4. Spectral response obtained through the bottom a-Si:H thick cell, with and without background bias light red $(650 \mathrm{~nm})$, blue $(450 \mathrm{~nm})$ and green $(550 \mathrm{~nm})$, incident power: $220 \mu \mathrm{W} \mathrm{cm}{ }^{-2}$. Background light entering through the top a-SiC:H thin cell. Light from the monochromator enter through the thick bottom cell with a power of $10 \mu \mathrm{W} \mathrm{cm}^{-2}$.

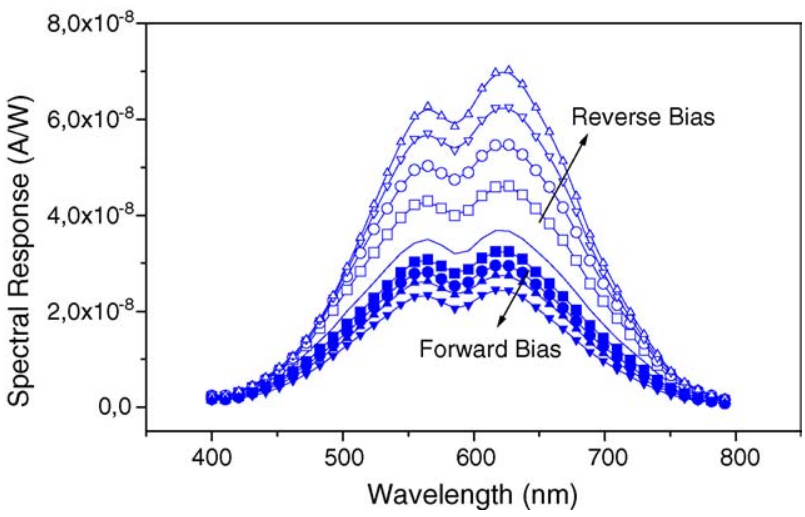

Fig. 5. Spectral response, at different applied bias (from -2 to $2 \mathrm{~V}$ ) obtained illuminating through the bottom a-Si:H thick cell, with background blue background bias light $(450 \mathrm{~nm})$ entering through the top a-SiC:H thin cel lwith incident power of $220 \mu \mathrm{W} \mathrm{cm}{ }^{-2}$. Light from the monochromator enter through the thick bottom cell with a power of $10 \mu \mathrm{W} \mathrm{cm}{ }^{-2}$.

ward bias reduce the responsivity of the structure, while the application of a reverse bias enhances it, and the difference between the SR profile with blue bias light and without bias light reach its maximum at values of the applied reverse bias above $2 \mathrm{~V}$. We can explain this different behaviour of the photocurrent by considering that carrier transport under red bias light condition is limited by the diffusion process at the internal recombination junction. While carrier transport under the blue bias light is drift dominated and sensible to variation of the external bias. The green case falls in a middle situation.

These different behaviours can be well observed in Fig. 6 , which reports the photocurrent produced by a pulsed red scanner (wavelength $650 \mathrm{~nm}$ ) shining on the bottom a-Si:H cell, while a bias light of different wavelength enters the device through the top a-SiC:H cell. The photocurrent without bias light, or with red bias light, does not vary with the applied external bias, while under blue and green bias light, respectively at forward and reverse applied bias, the photocurrent reduces to the corresponding one with no bias light. With the

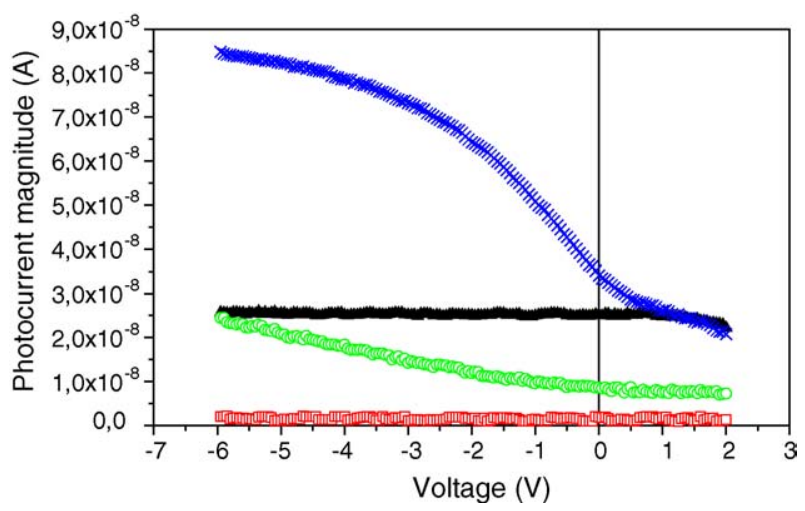

Fig. 6. Photocurrent produced a pulsed red scanner (wavelength $650 \mathrm{~nm}$, power flux $5.5 \mu \mathrm{W} \mathrm{cm}^{-2}$ ) shining on the bottom a-Si:H cell, while a bias light of different wavelength enters the device through the top a-SiC:H cell (red bias light: $650 \mathrm{~nm}$, green bias light $550 \mathrm{~nm}$, blue bias light: $450 \mathrm{~nm}$, power flux $50 \mu \mathrm{W} \mathrm{cm} \mathrm{cm}^{-2}$. 
application of a forward bias is possible to cancel out the blue part of the incident spectrum and detect the sum of the green and the red bias light contributions to the photocurrent. With the application of a reverse bias the device becomes blind to the green colour, and it is possible to detect the red and the blue contribution to the photocurrent, differentiated by opposite algebraic sign, leading to a complete RGB colour sensor. Applications of this structure as a colour laser scanned photodiode (CLSP) image sensor are presented at this same conference [6]

\section{Simulation results}

Based on the results about the internal electrical configuration of the device obtained with our numerical simulator ASCA [7], we present here an explanation of the device dynamics. This insight into the device physics permits a general understanding of the device behavior and gives some hints about future optimization of the device performance as a CLSP image sensor. For sake of simplicity in plot reading, we present only results about the internal configuration under blue and red light irradiation. The green light case is to be intended to fall in between the red and the blue ones.

Fig. 7 shows the different photogeneration rate profiles within the device under illumination through the top cell with different wavelengths, and the corresponding recombination rates. Because of the high optical gap in the a-SiC:H material (above $2.0 \mathrm{eV}$ ), photogeneration within the top cell is almost null under red light conditions (the simulator does not consider sub-band absorption). Being the top cell blind to the red radiation, and the photogeneration almost uniform within the bottom cell, we can assume that, under red light, the side chosen for illumination does not play any important role. This conclusion is also confirmed by the experimental $\mathrm{I}-\mathrm{V}$ characteristics reported in Figs. 2 and 3.

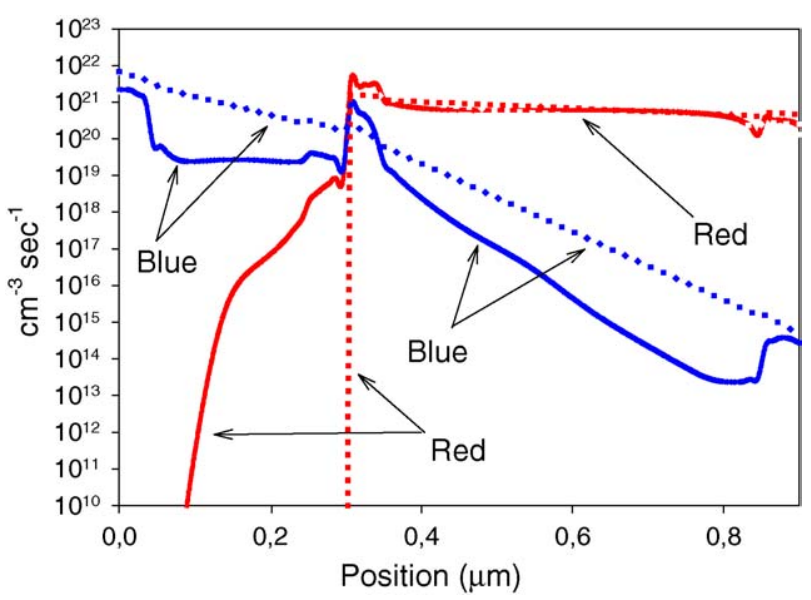

Fig. 7. Simulated photogeneration (dotted lines) and recombination rate (solid lines) profile within the structure in short-circuit condition for different light wavelength.
Under blue light radiation, the photogeneration process is mainly limited to the top cell, assuming low values within the bottom cell. The corresponding recombination rate profile reveals the nature of the transport mechanism within the device. While under blue light radiation the recombination rate remains constantly below the photogeneration one, under red light radiation, within the top cell (where there is no photogeneration) assumes low positive values due to the carrier diffusion through the internal recombination junction; within the bottom cell there is an accumulation of charge, and the recombination rate assumes a profile very close to the photogeneration one. Fig. 8 reports the corresponding simulated free carrier profiles. It is possible to observe here the different carriers profile distribution within the device under the different illumination conditions. The presence of carrier excess under red light condition in the top cell is to be related to carrier diffusion through the internal recombination junction. Being the resulting photocurrent value equal to the area delimited by the two profiles along the entire device, considering the redistribution of the carriers under red illumination limited by the diffusion process at the internal recombination junction, being moreover the well distributed recombination profile under blue illumination dependent on the external applied bias, it is expectable the observation of a strong dependence of the photocurrent from the applied bias under blue radiation (drift dominated transport mechanism) and a very slight dependence under red light (diffusion dominated transport mechanism). Finally, in Fig. 9 it is reported the simulated potential profiles within the structure for different wavelengths of the incident light. Under short circuit condition, the perturbation on the potential profile due to the incident light depends on the wavelength. In all the cases considered, and even under low power radiation, the potential barrier at the internal $n-p$ interface is reduced. In the absorber cell the potential profile become flat,

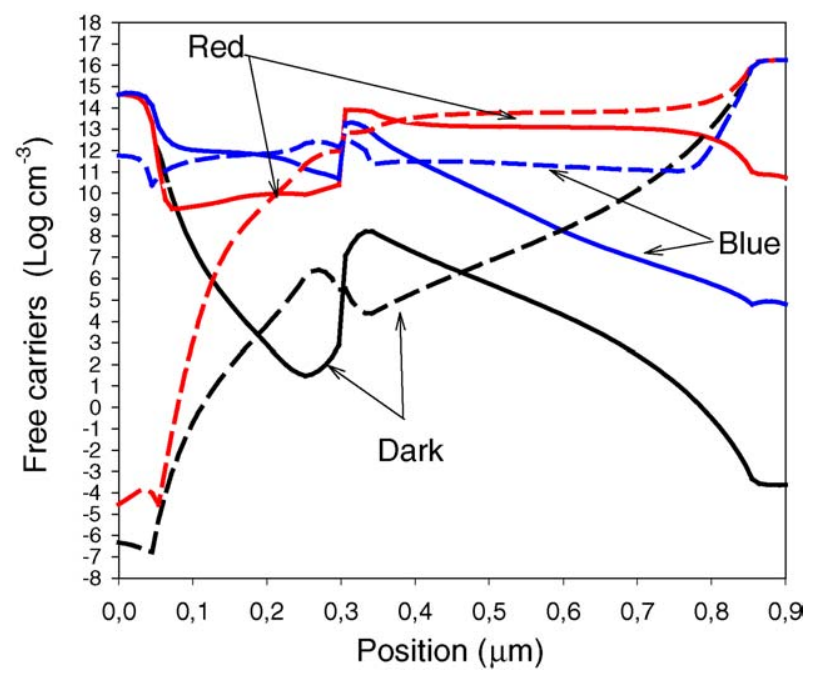

Fig. 8. Simulated free carriers profiles in short-circuit condition (dashed lines: electrons, solid lines: holes) within the structure for different wavelengths of the incident light. 


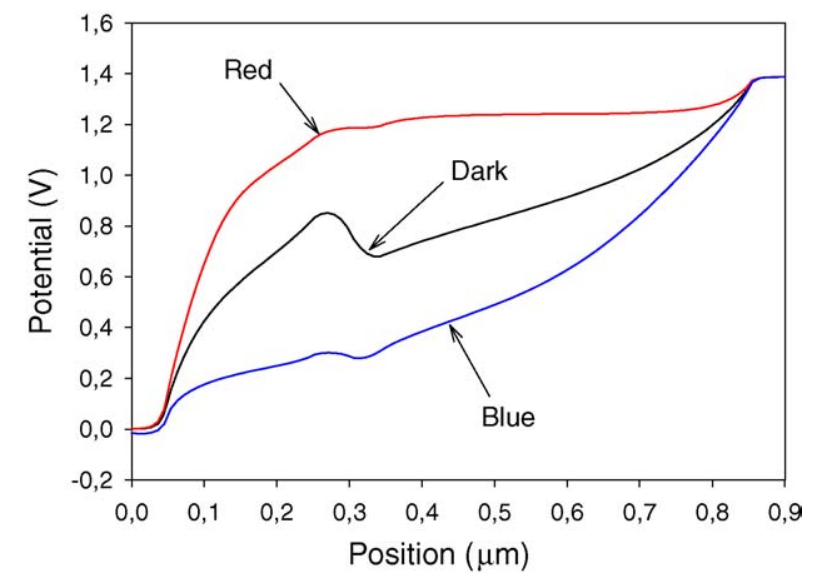

Fig. 9. Simulated potential profiles in short-circuit condition within the structure for different wavelengths of the incident light.

while the other cell suffers a reverse internal self-biasing to compensate the potential variation. We observe an opposite behavior under low energy radiations (red) and high energy ones (blue), due to the different penetration length of the light.

The photocurrent perturbation read through the LSP technique with a pulsed red laser incident at the bottom surface (see Fig. 6), must be interpreted as a direct dependence of the photocurrent on the incident power light in the case of a red image reading, and as a compensation of the reverse internal self-biasing of the bottom cell when the cell is illuminated with a blue image. Superposition of bias light and external applied bias can cancel out the blue and green photocurrent at a specific value of the applied bias that depends on the power density of both the image and scanner light. It remains a clear idea that with a strong effort in device calibration, this tandem heterostructure can be used for color recognition applications. Finally, while the detection of the blue color can be considered satisfactory, a speculative interpretation of the presented experimental results together with our simulation analysis, leads us to believe that optimization of this device can be obtained by reducing the thickness of the internal recombination junction (in order to enhance diffusion transport for red detection) and by increasing the intrinsic layer thickness of the bottom a$\mathrm{Si}: \mathrm{H}$ cell (in order to obtain a better detection of green color by enhancing the reverse internal self-biasing of the bottom cell).

\section{Conclusion}

A novel device structure based on a tandem structure (a-SiC:H:p/a-SiC:H/a-SiC:H:n/a-SiC:H:p/a-Si:H/a-Si:H:n) has been tested for a proper color separation process that takes advantage on the different filtering properties due to the different light penetration depth at different wavelengths a-Si:H and a-SiC:H. Experimental results about I-V characteristics and spectral response have been presented and explained in terms of drift/diffusion nature of the photocurrent by a recourse to a numerical simulation.

Forwarding the application of this tandem heterostructure for color recognition, detection of the blue color can be considered satisfactory. Device optimization for red detection can to be obtained by reducing the thickness of the internal recombination junction while by increasing the intrinsic layer of the bottom a-Si:H cell to is foreseen a better detection of the green color.

\section{References}

[1] M. Vieira, M. Fernandes, A. Fantoni, P. Louro, Y. Vygranenko, R. Schwarz, M. Schubert, LSP image sensors based on SiC heterostructures, Appl. Surf. Sci. 184 (2001) 471-476.

[2] M. Vieira, M. Fernandes, J. Martins, P. Louro, A. Maçarico, R. Schwarz, M. Schubert, Laser-scanned photodiode (LSP) for image detection, IEEE Sens. J. 1 (2001) 158-167.

[3] M. Vieira, M. Fernandes, A. Fantoni, P. Louro, R. Schwarz, A new CLSP sensor for Image recognition and color separation, Mat. Res. Soc. Symp. Proc. 715 (2002) A4.3.

[4] A. Fantoni, M. Fernandes, P. Louro, I. Rodrigues, M. Vieira, a$\mathrm{SiC}: \mathrm{H} / \mathrm{a}-\mathrm{Si}: \mathrm{H}$ tandem photodiods: a numerical simulation, Sens. Actuators A: Phys. 113 (2004) 324-328.

[5] G. Lavareda, C. Nunes de Carvalho, A. Amaral, J.P. Conde, M. Vieira, V. Chu, Properties of high growth rate amorphous silicon deposited by MC-RF-PECVD, Vacuum 64 (2002) 245-248.

[6] M. Vieira, M. Fernandes, P. Louro, A. Fantoni, G. Lavareda, C.Nunes de Carvalho, Y. Vygranenko, A two terminal optical signal and image processing $\mathrm{p}-\mathrm{i}-\mathrm{n} / \mathrm{p}-\mathrm{i}-\mathrm{n}$ image and colour sensor, Sens. Actuators A: Phys., in press, Corrected Proof, Available online 11 May 2005.

[7] A. Fantoni, M. Vieira, R. Martins, Simulation of hydrogenated amorphous and microcrystalline silicon optoelectronic devices, Math. Comput. Simul. 49 (1999) 381-401.

\section{Biographies}

Manuela Vieira was born in Lisbon, Portugal. In 1986 she received the Master of Science in Solid State Physics-Microelectronic and in 1993 the Ph.D. in Semiconductor Materials both from the New University of Lisbon. She is full professor in Electronics inside the department of Electronics Telecommunication and Computers (ISEL. Portugal) and the head of a Group in Applied Research in Microelectronic Optoelectronic and Sensors-GIAMOS. She has several scientific papers and 20 years of experience in the field of thin films and devices, her research activities have been mainly related to the development of optical sensors.

Miguel Fernandes was born in Portugal in 1970. He graduated in Physics and Materials Engineering by the Faculty of Sciences and Technology from the New University of Lisbon in 1995. Currently he is Assistant Professor in electronics department of ISEL, Lisbon, Portugal and investigator in the group of Applied Research in Microelectronic Optoelectronic and Sensors-GIAMOS in the same institution.

Paula Louro was born in Portugal in 1967. In 1990 she became researcher in EID Lisbon, Portugal, in the department of optoelectronics. She graduated in Physics by the Faculty of Sciences from the University of Lisbon in 1990. In 1995 she received the Master of Science in Material Engineering by the New University of Lisbon. Currently she is 
Assistant Professor in the electronics department of ISEL, Lisbon, Portugal, where she teaches Electronic and Semiconductor Physics. Her main research interest is in the field of amorphous semiconductor thin films and devices.

Alessandro Fantoni was born in Rome (Italy) in 1966. He received a university degree in applied mathematics from the University of Camerino,
Italy (1992), and a Ph.D. in Material Engineering/Micro and Optoelectronics from the New University of Lisbon, Portugal (1999). He actually teaches Semiconductor Physics in the Electronics, Telecommunications and Computer department on the Engineering Institute of Lisbon. His research interests are related to numerical analysis and simulation of micro and optoelectronic thin film devices. 